\title{
On the chemical ladder of esters
}

\section{Detection and formation of ethyl formate in the W51 e2 hot molecular core ${ }^{\star}$}

\author{
V. M. Rivilla ${ }^{1}$, M. T. Beltrán ${ }^{1}$, J. Martín-Pintado ${ }^{2}$, F. Fontani ${ }^{1}$, P. Caselli ${ }^{3}$, and R. Cesaroni ${ }^{1}$ \\ 1 Osservatorio Astrofisico di Arcetri, Largo Enrico Fermi 5, 50125 Firenze, Italy \\ e-mail: rivilla@arcetri.astro.it \\ 2 Centro de Astrobiología (CSIC-INTA), Ctra. de Torrejón a Ajalvir km 4, 28850 Torrejón de Ardoz, Madrid, Spain \\ 3 Max-Planck Institute for Extraterrestrial Physics, Giessenbachstrasse 1, 85748 Garching, Germany
}

Received 29 April 2016 / Accepted 30 October 2016

\begin{abstract}
Context. In recent years, the detection of organic molecules with increasing complexity and potential biological relevance is opening the possibility to understand the formation of the building blocks of life in the interstellar medium. One of the families of molecules of substantial astrobiological interest are the esters. The simplest ester, methyl formate $\left(\mathrm{CH}_{3} \mathrm{OCHO}\right)$, is rather abundant in star-forming regions. The next step in the chemical complexity of esters is ethyl formate, $\mathrm{C}_{2} \mathrm{H}_{5} \mathrm{OCHO}$. Despite the increase in sensitivity of current telescopes, the detection of complex molecules with more than ten atoms such as $\mathrm{C}_{2} \mathrm{H}_{5} \mathrm{OCHO}$ is still a challenge. Only two detections of this species have been reported so far, which strongly limits our understanding of how complex molecules are formed in the interstellar medium. New detections towards additional sources with a wide range of physical conditions are crucial to differentiate between competing chemical models based on dust grain surface and gas-phase chemistry.

Aims. We have searched for ethyl formate towards the W51 e2 hot molecular core, one of the most chemically rich sources in the Galaxy and one of the most promising regions to study prebiotic chemistry, especially after the recent discovery of the $\mathrm{P}-\mathrm{O}$ bond, key in the formation of DNA.

Methods. We have analyzed a spectral line survey towards the W51 e2 hot molecular core, which covers $44 \mathrm{GHz}$ in the 1,2 and $3 \mathrm{~mm}$ bands, carried out with the IRAM $30 \mathrm{~m}$ telescope.

Results. We report the detection of the trans and gauche conformers of ethyl formate. A local thermodynamic equilibrium analysis indicates that the excitation temperature is $78 \pm 10 \mathrm{~K}$ and that the two conformers have similar source-averaged column densities of $(2.0 \pm 0.3) \times 10^{-16} \mathrm{~cm}^{-2}$ and an abundance of $\sim 10^{-8}$. We compare for the first time the observed molecular abundances of ethyl formate with different competing chemical models based on grain surface and gas-phase chemistry.

Conclusions. We propose that grain-surface chemistry may have a dominant role in the formation of ethyl formate (and other complex organic molecules) in hot molecular cores, rather than reactions in the gas phase.
\end{abstract}

Key words. astrochemistry - astrobiology - line: identification - stars: formation - ISM: molecules - stars: massive

\section{Introduction}

The increase in sensitivity and bandwidth of current radiotelescopes allow us to detect molecules with increasing number of atoms in interstellar and circumstellar environments. Among the nearly 200 molecules already detected, $\sim 60$ are considered complex organic molecules (COMs), that is, they contain carbon and have six or more atoms. Since many of these molecules could play an important role in basic prebiotic chemistry, they are considered to be the building blocks of life. The study of the formation of COMs are crucial to understand how biochemistry could have emerged in the Universe. However, although our knowledge of the chemical complexity of the interstellar medium (ISM) is growing, our understanding about the synthesis of COMs is still very limited. Two general scenarios have been proposed for the formation of COMs: gas-phase chemistry triggered by the thermal evaporation of interstellar ices at high temperatures of $T>100 \mathrm{~K}$ was first invoked (e.g., Millar et al. 1991), while dust grain surface chemistry at lower temperatures $(10<T<80 \mathrm{~K})$ has been introduced in the last decade

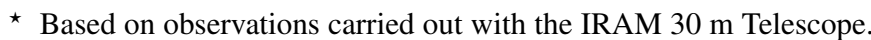
IRAM is supported by INSU/CNRS (France), MPG (Germany) and IGN (Spain).
}

(e.g., Garrod \& Herbst 2006). The detection of molecules with increasing complexity in different environments will help us to understand how COMs are formed in the ISM.

One of the families of COMs that attracts substantial astrobiological interest is that of esters, which are essential for many prebiotic processes. Some kind of esters, glycerides, are the basic constituents of human and animal fats and vegetable oils. Phosphoesters are key to the formation of the backbone of the genetic macromolecules ribonucleic acid (RNA) and deoxyribonucleic acid (DNA). The simplest representative of the ester family is the eight-atom molecule methyl formate $\left(\mathrm{CH}_{3} \mathrm{OCHO}\right.$, hereafter MF). Interestingly, this molecule is rather abundant in many high-mass and low-mass star-forming regions (e.g., Brown et al. 1975; Cazaux et al. 2003; Favre et al. 2011) and in the Galactic center (Requena-Torres et al. 2008). This makes the interstellar search for more complex esters very promising. The next step in ester complexity is the 11-atom molecule ethyl formate $\left(\mathrm{C}_{2} \mathrm{H}_{5} \mathrm{OCHO}\right.$, hereafter $\left.\mathrm{EF}\right)$. Despite the increase in sensitivity of current telescopes, this species has been detected so far only towards two hot molecular cores (SgrB2 N and Orion KL; Belloche et al. 2009; and Tercero et al. 2013, 2015), which clearly shows the difficulty of detecting molecules with such complexity. SgrB2 $\mathrm{N}$ and Orion KL are commonly adopted 
as templates for astrochemical studies because of their chemical richness. However, they are peculiar sources, and might not be fully representative of the chemical content of typical starforming regions in our galaxy. The SgrB2 $\mathrm{N}$ core (with a mass of $\sim 2.4 \times 10^{3} M_{\odot}$; Schmiedeke et al. 2016) is located in the Galactic center, where the physical and chemical conditions are unique, thus an extrapolation to other galactic star-forming regions is not straightforward. Moreover, although the astrochemical study of Orion KL is important due to its small distance (414 pc; Menten et al. 2007), this object cannot be considered a typical galactic hot core because of its relatively low mass ( $\sim 10 M_{\odot}$; Kurtz et al. 2000). Therefore, to better understand how COMs are formed in star-forming regions, more detections towards other hot cores are needed.

In this regard, the W51 star-forming region is a very promising target. Located at a distance of $5.1 \mathrm{kpc}$ (Xu et al. 2009) in the Sagittarius arm of our Galaxy, it harbors two compact radio sources (Scott 1978) known as e1 and e2, which are associated with hot molecular cores (Zhang \& Ho 1997; Zhang et al. 1998 ) with a mass $\sim 300 M_{\odot}$. These cores exhibit a rich chemistry with $\sim 50$ molecular species already detected (Liu et al. 2001; Ikeda et al. 2001; Remijan et al. 2002; Demyk et al. 2008; Kalenskii \& Johansson 2010). This chemical richness has encouraged several groups to carry out fruitless attempts to detect very complex prebiotic molecules, such as glycine $\left(\mathrm{NH}_{2} \mathrm{CH}_{2} \mathrm{COOH}\right.$, ten atoms; Snyder et al. 2005) or trans-ethyl methyl ether $\left(\mathrm{C}_{2} \mathrm{H}_{5} \mathrm{OCH}_{3}\right.$, twelve atoms; Carroll et al. 2015). Kalenskii \& Johansson (2010) marginally detected several complex species, being ethylene glycol $\left(\left(\mathrm{CH}_{2} \mathrm{OH}\right)_{2}\right.$, ten atoms) and EF (eleven atoms) among them. The recent confirmation of the presence of ethylene glycol towards W51 e2 by Lykke et al. (2015) opens up the possibility of also confirming the detection of EF in this region. It is remarkable that this source is now a main target for astrochemical prebiotic studies, after the first detection in a star-forming region of the $\mathrm{P}-\mathrm{O}$ bond, a key piece of the backbone of the RNA and DNA (Rivilla et al. 2016). For all these reasons, we have searched for EF towards the W51 starforming region.

$\mathrm{EF}$ is one of the largest molecules detected in the ISM but its formation is poorly known. Its millimeter rotational spectrum has been studied by Demaison (1984) and Medvedev et al. (2009). Only two conformers have been identified in experimental studies: i) the trans conformer, where the $\mathrm{C}-\mathrm{C}-\mathrm{O}-\mathrm{C}=\mathrm{O}$ chain forms a coplanar zigzag; and ii) the gauche conformer, with an out-of-plane rotation (to the left or to the right) of the ethyl group $\left(\mathrm{C}_{2} \mathrm{H}_{5}\right)$ around the $\mathrm{O}-\mathrm{C}$ bond. The ground state of the gauche conformer is $94 \pm 30 \mathrm{~K}$ higher in energy (Riveros \& Wilson 1967) than that of the trans conformer. The geometry of the conformers was studied by Peng (1995), and is presented in Fig. 1 of Medvedev et al. (2009).

In this paper, we use a spectral survey towards the W51 e2 hot molecular core, with a total bandwidth of $\sim 44 \mathrm{GHz}$, covering the 1, 2 and $3 \mathrm{~mm}$ bands, carried out with the IRAM $30 \mathrm{~m}$ telescope. We report the first detection of both conformers of EF towards the W51 e2 hot core, and discuss the implications of these observations on the formation of this complex species by confronting the observed molecular abundances with the predictions of the competing formation models based on grain-surface and gas-phase chemistry.

\section{Observations}

We have used data from different observational campaigns performed with the IRAM $30 \mathrm{~m}$ telescope at Pico Veleta (Spain)
Table 1. Summary of IRAM $30 \mathrm{~m}$ observations towards the W51 e1/e2 hot molecular core used in this work.

\begin{tabular}{cccc}
\hline \hline Date & $\begin{array}{c}\mathrm{RA}_{\text {J2000 }} \\
19^{\mathrm{h}} 23^{\mathrm{m}}\end{array}$ & $\begin{array}{c}\mathrm{Dec}_{\text {J2000 }} \\
14^{\circ} 30^{\prime}\end{array}$ & $\begin{array}{c}\text { Frequency coverage } \\
(\mathrm{GHz})\end{array}$ \\
\hline 2012 Apr. 25 & $43.90 \mathrm{~s}$ & $32.0^{\prime \prime}$ & $88.83-96.60$ \\
& & & $104.51-112.29$ \\
\hline 2012 Dec. 13 & $43.90 \mathrm{~s}$ & $34.8^{\prime \prime}$ & $99.70-106.30$ \\
& & & $238.20-245.98$ \\
\hline 2015 Dec. 9 & $43.90 \mathrm{~s}$ & $32.0^{\prime \prime}$ & $134.47-142.25$ \\
& & & $150.15-157.90$ \\
\hline
\end{tabular}

on April and December ${ }^{1} 2012$ and December 2015. All campaigns used the Eight Mixer Receiver (EMIR) coupled with the Fast Fourier Transform Spectrometer (FTS), which provided a spectral resolution of $0.195 \mathrm{kHz}$. The total spectral bandwidth covered is $\sim 44 \mathrm{GHz}$, in the ranges $88.83-96.60,99.70-112.29$, $134.47-142.25,150.15-157.90$ and $238.20-245.98 \mathrm{GHz}$. The spectra were exported from the software package CLASS of GILDAS $^{2}$ to MADCUBAIJ ${ }^{3}$ (see e.g., Rivilla et al. 2017), which was used for the line identification and analysis.

The line intensity of the IRAM $30 \mathrm{~m}$ spectra was converted to the main beam temperature $T_{\mathrm{mb}}$ scale, using the efficiencies provided by IRAM ${ }^{4}$. The half-power beam widths of the observations can be estimated using the expression $\theta_{\text {beam }}$ [arcsec] $=$ $2460 / v(\mathrm{GHz})$, and are in the range $10^{\prime \prime}-27^{\prime \prime}$. All the observed spectra were smoothed to a velocity resolution of $1.5 \mathrm{~km} \mathrm{~s}^{-1}$.

The position of the 2012 December observations $\left(19^{\mathrm{h}} 23^{\mathrm{m}} 43.9^{\mathrm{s}}, 14^{\circ} 30^{\prime} 34.8^{\prime \prime}\right)$ is shifted by $2.8^{\prime \prime}$ in declination with respect to the other two epochs $\left(19^{\mathrm{h}} 23^{\mathrm{m}} 43.9^{\mathrm{s}}\right.$, $\left.14^{\circ} 30^{\prime} 32.0^{\prime \prime}\right)$. We have checked that this shift in position does not significantly affect the line intensities by comparing the April and December 2012 spectra in the range where they overlap: 104.51-106.30 GHz. The line intensities of the December data are only a factor $\sim 1.1$ higher than those of the April data, within the expected calibration uncertainties.

\section{Results}

The observed spectrum contain multiple transitions of both conformers of EF. We have used MADCUBAIJ to identify the lines and model the expected line profiles for the two conformers of EF assuming local thermodynamic equilibrium (LTE) conditions. For the analysis we have used the spectroscopic parameters from the Jet Propulsion Laboratory (JPL) molecular catalog 5 . In Fig. 1 we show 42 unblended or slightly blended transitions of the trans conformer of EF at 2 and $3 \mathrm{~mm}$ (see also Table 2). The $1 \mathrm{~mm}$ spectra exhibit a very high density of molecular lines, preventing the identification of any unblended EF transition.

We have also identified multiple transitions of other molecules that have been proposed to be chemically related to EF: the simplest ester methyl formate $\left(\mathrm{CH}_{3} \mathrm{OCHO}\right.$, hereafter MF), ethanol $\left(\mathrm{C}_{2} \mathrm{H}_{5} \mathrm{OH}\right.$, hereafter ET), and formic acid $(\mathrm{HCOOH})$.

\footnotetext{
1 The 2012 December data were obtained from Lykke et al. (2015).

2 http://wWw . iram. fr/IRAMFR/GILDAS

3 Madrid Data Cube Analysis on ImageJ is a software developed in the Center of Astrobiology (Madrid, INTA-CSIC) to visualize and analyze single spectra and datacubes (Martín et al., in prep.).

4 http://WWW . iram. es/IRAMES/mainWiki/

Iram30mEfficiencies

5 http://spec.jpl.nasa.gov/
} 
V. M. Rivilla et al.: Detection and formation of ethyl formate in the W51 e2 hot molecular core
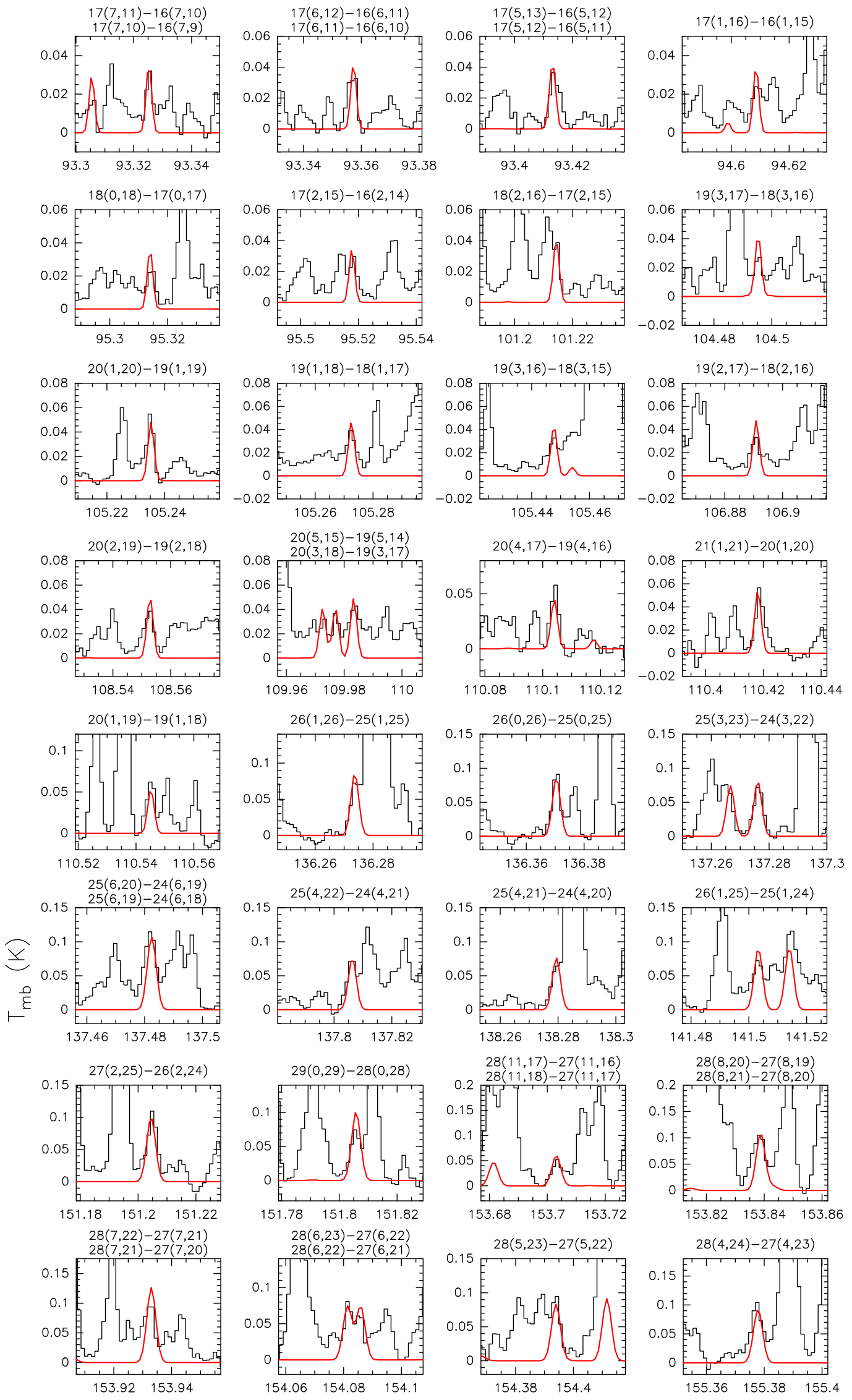

Frequency $(\mathrm{GHz})$

Fig. 1. Unblended or slightly blended transitions of the trans conformer of EF towards the W51 e2 hot core (see Table 2). We have overplotted in red the LTE synthetic spectrum obtained with MADCUBAIJ assuming a temperature of $78 \mathrm{~K}$, a column density of $2 \times 10^{16} \mathrm{~cm}^{-2}$ and a source size of $2.4^{\prime \prime}$. 
Table 2. Unblended or slightly blended transitions of the trans and gauche conformers of EF detected towards the W51 e2 hot molecular core.

\begin{tabular}{|c|c|c|c|}
\hline $\begin{array}{c}\text { Frequency } \\
\quad(\mathrm{GHz})\end{array}$ & Transition & $\begin{array}{l}E_{\text {up }} \\
(\mathrm{K})\end{array}$ & $\underset{\left(\mathrm{K} \mathrm{km} \mathrm{s}^{-1}\right)^{a}}{T_{\mathrm{mb}} \Delta v}$ \\
\hline \multicolumn{4}{|c|}{ Trans conformer } \\
\hline 93.3247 & $17(7,11)-16(7,10)$ & 76 & $0.13 \pm 0.03$ \\
\hline 93.3247 & $17(7,10)-16(7,9)$ & 76 & $0.13 \pm 0.03$ \\
\hline 93.356781 & $17(6,12)-16(6,11)$ & 66 & $0.16 \pm 0.04$ \\
\hline 93.356798 & $17(6,11)-16(6,10)$ & 66 & $0.16 \pm 0.04$ \\
\hline 93.41211 & $17(5,13)-16(5,12)$ & 58 & $0.18 \pm 0.04$ \\
\hline 93.413115 & $17(5,12)-16(5,11)$ & 58 & $0.18 \pm 0.04$ \\
\hline 94.608041 & $17(1,16)-16(1,15)$ & 42 & $0.26 \pm 0.06$ \\
\hline 95.313367 & $18(0,18)-17(0,17)$ & 44 & $0.27 \pm 0.06$ \\
\hline 95.517215 & $17(2,15)-16(2,14)$ & 44 & $0.25 \pm 0.06$ \\
\hline 101.213864 & $18(2,16)-17(2,15)$ & 49 & $0.30 \pm 0.07$ \\
\hline 104.49469 & $19(3,17)-18(3,16)$ & 57 & $0.31 \pm 0.07$ \\
\hline 105.23465 & $20(1,20)-19(1,19)$ & 54 & $0.35 \pm 0.08$ \\
\hline 105.27195 & $19(1,18)-18(1,17)$ & 52 & $0.34 \pm 0.08$ \\
\hline 105.44706 & $19(3,16)-18(3,15)$ & 57 & $0.32 \pm 0.07$ \\
\hline 106.89024 & $19(2,17)-18(2,16)$ & 54 & $0.35 \pm 0.08$ \\
\hline 108.55223 & $20(2,19)-19(2,18)$ & 58 & $0.37 \pm 0.08$ \\
\hline 109.976377 & $20(5,15)-19(5,14)$ & 73 & $0.30 \pm 0.06$ \\
\hline 109.982644 & $20(3,18)-19(3,17)$ & 62 & $0.36 \pm 0.08$ \\
\hline 110.103357 & $20(4,17)-19(4,16)$ & 67 & $0.33 \pm 0.07$ \\
\hline 110.417497 & $21(1,21)-20(1,20)$ & 59 & $0.40 \pm 0.08$ \\
\hline 110.544519 & $20(1,19)-19(1,18)$ & 57 & $0.39 \pm 0.08$ \\
\hline 136.272898 & $26(1,26)-25(1,25)$ & 90 & $0.6 \pm 0.1$ \\
\hline 136.36963 & $26(0,26)-25(0,25)$ & 89 & $0.6 \pm 0.1$ \\
\hline 137.275291 & $25(3,23)-24(3,22)$ & 92 & $0.6 \pm 0.1$ \\
\hline 137.48096 & $25(6,20)-24(6,19)$ & 112 & $0.45 \pm 0.07$ \\
\hline 137.482273 & $25(6,19)-24(6,18)$ & 112 & $0.47 \pm 0.07$ \\
\hline 137.805525 & $25(4,22)-24(4,21)$ & 97 & $0.56 \pm 0.09$ \\
\hline 138.27859 & $25(4,21)-24(4,20)$ & 97 & $0.56 \pm 0.09$ \\
\hline 141.50245 & $26(1,25)-25(1,24)$ & 94 & $0.7 \pm 0.1$ \\
\hline 151.20362 & $27(2,25)-26(2,24)$ & 104 & $0.7 \pm 0.1$ \\
\hline 151.80493 & $29(0,29)-28(0,28)$ & 111 & $0.7 \pm 0.1$ \\
\hline 153.70219 & $28(11,17)-27(11,16)$ & 194 & $0.22 \pm 0.03$ \\
\hline 153.70219 & $28(11,18)-27(11,17)$ & 194 & $0.22 \pm 0.03$ \\
\hline 153.83787 & $28(8,20)-27(8,19)$ & 153 & $0.40 \pm 0.05$ \\
\hline 153.83787 & $28(8,21)-27(8,20)$ & 153 & $0.40 \pm 0.05$ \\
\hline 153.931996 & $28(7,22)-27(7,21)$ & 142 & $0.47 \pm 0.06$ \\
\hline 153.932149 & $28(7,21)-27(7,20)$ & 142 & $0.47 \pm 0.06$ \\
\hline 154.08046 & $28(6,23)-27(6,22)$ & 133 & $0.54 \pm 0.07$ \\
\hline 154.08500 & $28(6,22)-27(6,21)$ & 133 & $0.54 \pm 0.07$ \\
\hline 154.39306 & $28(5,23)-27(5,22)$ & 125 & $0.61 \pm 0.09$ \\
\hline 155.37695 & $28(4,24)-27(4,23)$ & 119 & $0.7 \pm 0.1$ \\
\hline 156.91332 & $30(1,30)-29(1,29)$ & 118 & $0.8 \pm 0.1$ \\
\hline \multicolumn{4}{|c|}{ Gauche conformer } \\
\hline 141.56623 & $20(4,17)-19(4,16)$ & 173 & $0.11 \pm 0.02$ \\
\hline 156.01357 & $22(10,13)-21(10,12)$ & 213 & $0.08 \pm 0.01$ \\
\hline 156.01358 & $22(10,12)-21(10,11)$ & 213 & $0.08 \pm 0.01$ \\
\hline 157.37580 & $22(3,19)-21(3,18)$ & 187 & $0.14 \pm 0.02$ \\
\hline
\end{tabular}

Notes. ${ }^{(a)}$ From the LTE model fit (see text).

The beams of the observations $\left(10^{\prime \prime}-27^{\prime \prime}\right)$ include two hot molecular cores, W51 e1 and e2, which have different radial velocities of $\sim 59 \mathrm{~km} \mathrm{~s}^{-1}$ and $\sim 55 \mathrm{~km} \mathrm{~s}^{-1}$, respectively (Zhang et al. 1998). To infer which of the two cores is dominating the detected molecular emission we have used the radial velocities of the lines. The velocities of different COMs studied here are in the range $55.2-56.4 \mathrm{~km} \mathrm{~s}^{-1}$, and hence they likely arise mainly from e2. In agreement with this interpretation, Kalenskii \& Johansson (2010) concluded that O-bearing species are mainly associated with core e2, while N-bearing species are associated with core e1. Therefore, we have considered that the molecular emission of the molecules discussed here mainly arises from W51 e2.

We have fitted the 42 transitions of the trans conformer of EF nearly free of contamination (Fig. 1 and Table 2) to obtain the physical parameters of the emission. MADCUBAIJ takes into account five different parameters to model the expected LTE line profiles: source-averaged column density of the molecule $(N)$, excitation temperature $\left(T_{\mathrm{ex}}\right)$, linewidth $(\Delta v)$, peak velocity $(v)$ and source angular diameter $(\theta)$. The procedure used to fit the data was as follows: (i) firstly, we fixed the size of the molecular emission of W51 e2 to $2.4^{\prime \prime}$ (as derived by Zhang et al. 1998) and applied the beam dilution factor for each transition accordingly; (ii) secondly, we fixed the velocity and the linewidth to the values that reproduce well the observed spectral profile of the unblended lines; and (iii) thirdly, leaving $N$ and $T_{\text {ex }}$ as free parameters, we used the AUTOFIT tool of MADCUBAIJ, which resolves the radiative transfer equation for a uniform slab and provides the best non-linear least-squared fit using the Levenberg-Marquardt algorithm. We have found the best fit for $T_{\text {ex }}=78 \pm 10 \mathrm{~K}$ and $N=(2.0 \pm 0.3) \times 10^{16} \mathrm{~cm}^{-2}$ (Table 3). To derive the molecular abundance, we have used an $\mathrm{H}_{2}$ column density of $2 \times 10^{24} \mathrm{~cm}^{-2}$ (Ginsburg, priv. comm.). The $\mathrm{H}_{2}$ column density has been calculated from the continuum flux at $1 \mathrm{~mm}$, recently observed with ALMA, over a region of $2^{\prime \prime}$ size, similar to that of the COMs emission assumed in this work, and assuming a temperature of $100 \mathrm{~K}$, similar to the excitation temperature derived for the COMs (see Table 3). We have obtained an abundance of EF is $1 \times 10^{-8}$. The line opacities derived from the fits are $\tau \leq 0.07$, so we can conclude that the EF emission is optically thin. We stress that all the predicted transitions of the trans conformer of EF with line intensities $>5 \sigma$ are compatible with the observed spectrum.

In the case of the gauche conformer, the predicted LTE line intensities are generally lower than those of the trans conformer at 2 and $3 \mathrm{~mm}$, which makes its identification more difficult. In Fig. 2 we show some selected transitions of the gauche conformer. The LTE spectrum assuming the same physical parameters used for the trans conformer reproduces very well the observed spectral features, in particular the transitions at $141.56623 \mathrm{GHz}$ and the two hyperfine transitions at 156.0135 GHz (see Fig. 2 and Table 2; these transitions were already identified in Orion by Tercero et al. 2013; see their Fig. 4). All the predicted transitions of the gauche conformer are compatible with the observed spectra. Therefore, we conclude that the gauche conformer is also detected in W51 e2, with similar abundance to that of the trans conformer, as found by Tercero et al. (2013) in Orion. Belloche et al. (2009) detected the trans conformer in SgrB2 $\mathrm{N}$ and reported only upper limits for the gauche conformer, although they do not exclude similar abundances for both conformers.

The gauche conformer has two possible orientations of the ethyl group $\left(\mathrm{C}_{2} \mathrm{H}_{5}\right)$ around the $\mathrm{O}-\mathrm{C}$ bond (see Medvedev et al. 2009). This implies that the relative abundance of both conformers in thermodynamic equilibrium is $N_{\mathrm{g}} / N_{\mathrm{t}}=2 \mathrm{e}^{-\Delta E / T_{\text {kin }}}$, where $\mathrm{g}$ and $\mathrm{t}$ refer to the gauche and trans conformers, respectively, $T_{\text {kin }}$ is the kinetic temperature of the gas and $\Delta E$ is the energy difference between the two ground states of the two conformers. The factor of two accounts for the two degenerated gauche conformers. Riveros \& Wilson (1967) found that this difference is in 
Table 3. Physical parameters of the molecules obtained from the LTE analysis of W51, and comparison with SgrB2 N.

\begin{tabular}{ccccccccc}
\hline \hline Molecule & $N\left(\times 10^{16} \mathrm{~cm}^{-2}\right)$ & \multicolumn{2}{c}{$X\left(10^{-8}\right)$} & \multicolumn{2}{c}{$T_{\text {ex }}(\mathrm{K})$} & \multicolumn{2}{c}{$\Delta v\left(\mathrm{~km} \mathrm{~s}^{-1}\right)$} \\
& W51 & SgrB2 & W51 & SgrB2 N & W51 & SgrB2 N & W51 & SgrB2 N \\
\hline $\mathrm{C}_{2} \mathrm{H}_{5} \mathrm{OCHO}(\mathrm{EF})$ & $2.0 \pm 0.3$ & 5.4 & 1.0 & 0.36 & $78 \pm 10$ & 100 & 7.0 & 7.0 \\
$\mathrm{C}_{2} \mathrm{H}_{5} \mathrm{OH}(\mathrm{ET})$ & $15.8 \pm 0.4$ & 84 & 7.9 & 5.7 & $95 \pm 3$ & 100 & 6.9 & 8.0 \\
$\mathrm{CH}_{3} \mathrm{OCHO}(\mathrm{MF})$ & $50.1 \pm 0.6$ & 45 & 25 & 3.5 & $112 \pm 3$ & 80 & 6.9 & 7.2 \\
$\mathrm{t}-\mathrm{HCOOH}$ & $1.6 \pm 0.8$ & 1.5 & 0.8 & 0.13 & $18 \pm 3$ & 70 & 7.1 & 8.0 \\
\hline
\end{tabular}
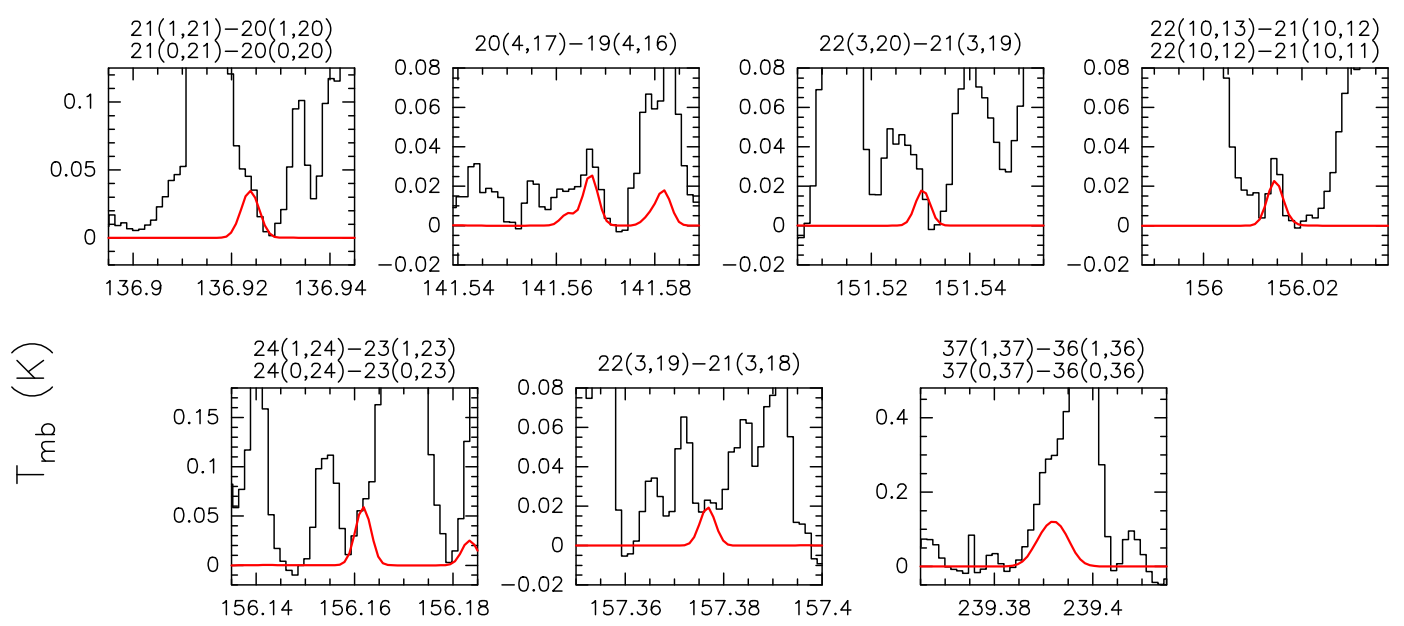

$$
\text { Frequency }(\mathrm{GHz})
$$

Fig. 2. Selected transitions of the gauche conformer of EF towards the W51 e2 hot core. We have overplotted in red the LTE synthetic spectrum obtained with MADCUBAIJ assuming the same physical parameters as the trans conformer: $T_{\mathrm{ex}}=78 \mathrm{~K}, N=2.0 \times 10^{16} \mathrm{~cm}^{-2}$ and a source size of $2.4^{\prime \prime}$.

the range $64-124 \mathrm{~K}$. If both conformers are equally abundant, as suggested by the W51 e2 and Orion data, this would imply that the kinetic temperature is in the range $T_{\text {kin }}=93-178 \mathrm{~K}$. If we assume that EF is thermalized, then $T_{\text {kin }} \simeq T_{\text {ex }}$. The excitation temperature derived for $\mathrm{EF}(78 \pm 10 \mathrm{~K}$; Table 3$)$ is slightly lower than the range of $T_{\text {kin. }}$. This is probably because the transitions of the trans conformer (with energies in the range 42-194 K) are tracing colder gas than the higher-energy transitions of the gauche conformer (see Table 2). In any case, we stress that the observed spectra support the fact that the two conformers share a similar abundance.

We have also fitted the emission of MF and ET with the same procedure used for EF. Moreover, we have also fitted the emission of the more stable conformer of $\mathrm{HCOOH}$ (trans- $\mathrm{HCOOH}$, hereafter $\mathrm{t}-\mathrm{HCOOH}$ ), which has been suggested as a key species to form COMs (Taquet et al. 2016; see Sect. 4). In Appendix A we show some selected unblended transitions of MF, ET and $\mathrm{t}-\mathrm{HCOOH}$. In the case of $\mathrm{t}-\mathrm{HCOOH}$, since this molecule usually traces gas at lower temperatures (see e.g., Bisschop et al. 2007), one would expect a larger emitting region than that of COMs. For this reason, we have left the source size as a free parameter in the AUTOFIT tool of MADCUBAIJ. The derived physical parameters are summarized in Table 3. For t-HCOOH we have found a lower temperature, $18 \pm 3 \mathrm{~K}$, as expected and a source size of $3.2^{\prime \prime}$, only slightly larger than that assumed for the COMs. We note that if the size of the emission of $\mathrm{HCOOH}$ is larger than this value, then the value of the column density presented in Table 3 should be considered as an upper limit.

We have also searched for the more excited cis conformer of $\mathrm{HCOOH}$, which has been proposed to be formed at the surface of cold ices (Ioppolo et al. 2011), but we have not detected any transition. We have obtained an upper limit for the column density of $\sim 10^{15} \mathrm{~cm}^{-2}$ (more than an order of magnitude less abundant than $\mathrm{t}-\mathrm{HCOOH})$, considering the same temperature, velocity, linewidth and source size than that of $\mathrm{t}-\mathrm{HCOOH}$.

\section{Discussion: surface or gas-phase chemistry?}

Only a very few COMs with more than ten atoms have been detected in the ISM. In particular, EF (eleven atoms) has been reported so far only towards three sources: W51 e2 (this work), SgrB2 N (Belloche et al. 2009) and Orion (Tercero et al. 2013, 2015). Due to the limited detection of EF in the ISM, it was included only in one chemical model based on grain-surface chemistry (Garrod et al. 2008). This model was considered by Belloche et al. (2009) to explain the formation of EF in SgrB2 N. Obviously, due to the lack of alternative models, these authors did not discuss other possible formation routes for this species. For Orion, Tercero et al. (2013) used an energy argument based on gas-phase chemistry to explain the similar abundances of the two EF conformers. However, they did not compare the observed molecular abundances with any chemical model. Only very recently, a new gas-phase chemical network has included the formation of EF (Taquet et al. 2016). This opens up the possibility of confronting the observed molecular abundances against the predictions of competing models: one based on dust-grain chemistry (Garrod et al. 2008), and the other based on gas-phase chemistry (Taquet et al. 2016).

\subsection{Gas-phase formation}

In the following we will refer to the chemical reactions shown in Table 4 and Fig. 3. Charnley et al. (1995) proposed a chemical pathway through gas-phase reactions starting with the 
Table 4. Gas-phase and grain-surface chemical routes proposed in the literature for the formation of EF.

\begin{tabular}{|c|c|c|}
\hline \multicolumn{3}{|c|}{ Gas-phase chemistry } \\
\hline$(1)$ & $\mathrm{C}_{2} \mathrm{H}_{5} \mathrm{OH}(\mathrm{ET})+\mathrm{H}^{+} \longrightarrow \mathrm{C}_{2} \mathrm{H}_{5} \mathrm{OH}_{2}^{+}$ & Charnley et al. (1995) \\
\hline$(2 a)$ & $\mathrm{C}_{2} \mathrm{H}_{5} \mathrm{OH}_{2}^{+}\left(\mathrm{ET}^{+}\right)+\mathrm{H}_{2} \mathrm{CO} \longrightarrow \mathrm{HC}(\mathrm{OH}) \mathrm{OC}_{2} \mathrm{H}_{5}^{+}+\mathrm{H}_{2}$ & Charnley et al. (1995) \\
\hline$(2 b)$ & $\mathrm{HCOHOC}_{2} \mathrm{H}_{5}^{+}+\mathrm{e}^{-} \longrightarrow \mathrm{C}_{2} \mathrm{H}_{5} \mathrm{OCHO}(\mathrm{EF})+\mathrm{H}$ & \\
\hline (3a) & $\mathrm{C}_{2} \mathrm{H}_{5} \mathrm{OH}_{2}^{+}\left(\mathrm{ET}^{+}\right)+\mathrm{HCOOH} \longrightarrow \mathrm{HC}(\mathrm{OH}) \mathrm{OC}_{2} \mathrm{H}_{5}^{+}+\mathrm{H}_{2} \mathrm{O}$ & Taquet et al. (2016) \\
\hline$(3 b)$ & $\mathrm{HC}(\mathrm{OH}) \mathrm{OC}_{2} \mathrm{H}_{5}^{+}+\mathrm{NH}_{3} \longrightarrow \mathrm{C}_{2} \mathrm{H}_{5} \mathrm{OCHO}(\mathrm{EF})+\mathrm{NH}_{4}^{+}$ & \\
\hline \multicolumn{3}{|c|}{ Grain-surface chemistry } \\
\hline (4a) & $\mathrm{HCO}+2 \mathrm{H} \rightarrow \mathrm{CH}_{3} \mathrm{O}$ & Garrod et al. (2008) \\
\hline (4b) & $\mathrm{CH}_{3} \mathrm{O}+\mathrm{HCO} \longrightarrow \mathrm{CH}_{3} \mathrm{OCHO}(\mathrm{MF})$ & \& Belloche et al. (2009) \\
\hline$(4 c)$ & $\mathrm{CH}_{3} \mathrm{OCHO}(\mathrm{MF})+h v \longrightarrow \mathrm{CH}_{2} \mathrm{OCHO}$ & \\
\hline$(4 d)$ & $\mathrm{CH}_{2} \mathrm{OCHO}+\mathrm{CH}_{3} \longrightarrow \mathrm{C}_{2} \mathrm{H}_{5} \mathrm{OCHO}(\mathrm{EF})$ & \\
\hline (5a) & $\mathrm{HCO}+2 \mathrm{H} \longrightarrow \mathrm{CH}_{2} \mathrm{OH}$ & Garrod et al. (2008) \\
\hline$(5 b)$ & $\mathrm{CH}_{2} \mathrm{OH}+\mathrm{CH}_{3} \longrightarrow \mathrm{C}_{2} \mathrm{H}_{5} \mathrm{OH}(\mathrm{ET})$ & \& Belloche et al. (2009) \\
\hline$(5 c)$ & $\mathrm{C}_{2} \mathrm{H}_{5} \mathrm{OH}(\mathrm{ET})+h v \longrightarrow \mathrm{C}_{2} \mathrm{H}_{5} \mathrm{O}$ & \\
\hline$(5 \mathrm{~d})$ & $\mathrm{C}_{2} \mathrm{H}_{5} \mathrm{O}+\mathrm{HCO} \longrightarrow \mathrm{C}_{2} \mathrm{H}_{5} \mathrm{OCHO}(\mathrm{EF})$ & \\
\hline
\end{tabular}

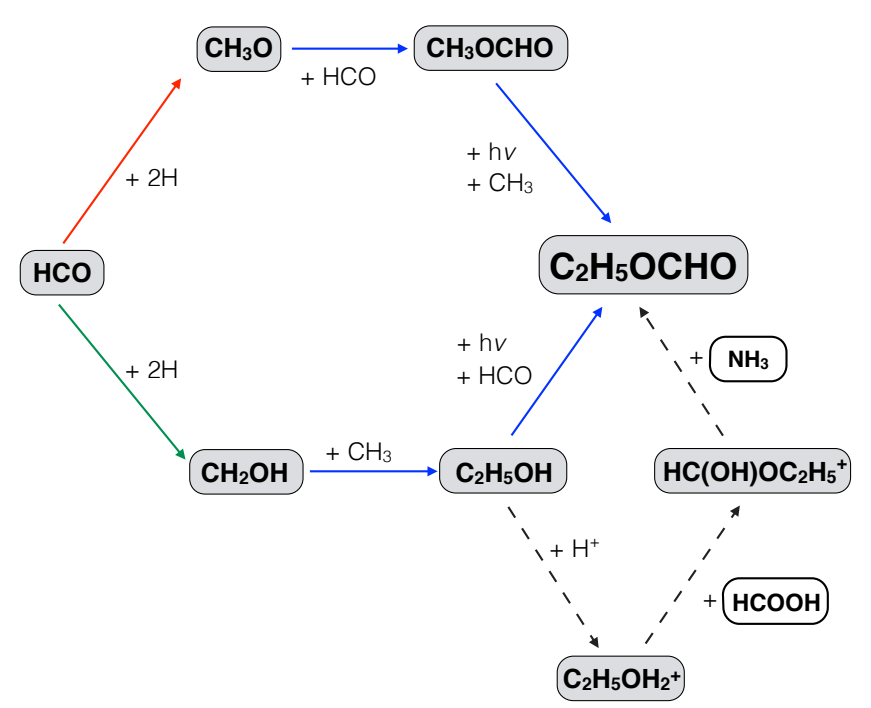

Fig. 3. Proposed formation routes for ethyl formate. Solid arrows indicate chemical reaction on grain surface, while dashed arrows correspond to gas-phase reactions. The references for the different pathways are: Tielens \& Whittet (1997), Beltrán et al. (2009), Woods et al. (2012; red arrow); Hama \& Watanabe (2013; green arrow); Garrod et al. (2008), Belloche et al. (2009; blue arrow); and Taquet et al. (2016; dashed black arrows).

protonation of ET, followed by a reaction with $\mathrm{H}_{2} \mathrm{CO}$ and subsequent recombination (reactions $1,2 \mathrm{a}$ and $2 \mathrm{~b}$ ). According to their model, this gas-phase route is able to produce EF abundances of $\sim 10^{-10}$ in $10^{4}-10^{5} \mathrm{yr}$, comparable to the timescales of hot cores (Fontani et al. 2007). The predicted abundance of $\mathrm{EF}$ is however more than one or two orders of magnitude lower than that estimated in W51 e2 and SgrB2 N, which suggests that this gas-phase route is not efficient enough to explain the observed abundance of EF. Moreover, reactions $2 \mathrm{a}$ and $2 \mathrm{~b}$ have not been studied yet in detail in the laboratory. An equivalent gasphase chemical pathway to form $\mathrm{MF}$, that is, the protonation of methanol $\left(\mathrm{CH}_{3} \mathrm{OH}\right)$ followed by a reaction with $\mathrm{H}_{2} \mathrm{CO}$, has also been proved inefficient by quantum theoretical calculations and laboratory experiments (Horn et al. 2004). This suggests that the reaction of protonated ET with $\mathrm{H}_{2} \mathrm{CO}$ (reaction 2a) might also be inefficient to form EF. We encourage the developement of new laboratory experiments to confirm this. Additional works (Geppert et al. 2006; Vigren et al. 2010; Hamberg et al. 2010a,b) showed that electron recombination reactions are mostly dissociative, which makes reaction $2 b$ unlikely.

Very recently, gas-phase chemistry has been reinvoked to explain the formation of COMs. New gas-phase formation routes based on laboratory experiments have been proposed to explain the formation of MF (Balucani et al. 2015). In the case of EF, the new theoretical chemical network by Taquet et al. (2016) indicates that the combination of protonated ET with $\mathrm{HCOOH}$ and $\mathrm{NH}_{3}$ (reactions $3 \mathrm{a}$ and $3 \mathrm{~b}$ ) are able to increase the EF abundance by more than one order of magnitude, making this route a promising way to form EF. Indeed, this gas-phase model is able to predict an abundance of EF of $10^{-9}-10^{-8}$ (Taquet et al. 2016), similar to that measured (Table 3 ). In this scenario, the presence of enough $\mathrm{HCOOH}$ and $\mathrm{NH}_{3}$ is key to allow the formation of EF via the chemical reactions $3 \mathrm{a}$ and $3 \mathrm{~b}$. According to this model, to achieve the observed molecular abundances of $\mathrm{EF},(0.36-1) \times 10^{-8}$, the reqired abundances of $\mathrm{HCOOH}$ and $\mathrm{NH}_{3}$ are of a few $10^{-7}$ and $\sim 10^{-6}$, respectively (Fig. 6 of Taquet et al. 2016). However, we have estimated that the abundance of $\mathrm{HCOOH}$ in W51 e2 is $\sim 10^{-8}$, while Belloche et al. (2009) found a value of $1.3 \times 10^{-9}$ towards SgrB2 N. These abundances are lower than those required for the gas-phase model by a factor $>50$.

Regarding $\mathrm{NH}_{3}$, Zhang \& Ho (1997) derived a column density of $\sim 10^{17} \mathrm{~cm}^{-2}$, which implies an abundance of $5 \times 10^{-8}$, which is also a factor 50 lower than that required by the gasphase model. Therefore, we conclude that these massive starforming regions do not have high enough abundance of $\mathrm{HCOOH}$ and $\mathrm{NH}_{3}$ to form efficiently $\mathrm{EF}$ and other COMs via the proposed gas-phase reactions.

\subsection{Grain-surface formation}

The lack of viable gas-phase reactions to form MF and EF (and other COMs) triggered in the past years the development of models based on grain-surface chemistry. Garrod et al. (2008) proposed that EF can be formed from the combination of simpler radicals, such as $\mathrm{HCO}, \mathrm{CH}_{3} \mathrm{O}$ and $\mathrm{CH}_{3}$ (reactions 4 and 5). $\mathrm{MF}$ is the primary source of precursor radicals of EF via photodissociation (reaction 4c). Once MF is desorbed from grains, then ET is the main source of precursor radicals (reaction 5c). The grainsurface model by Garrod et al. (2008) predicts an abundance of EF of $2.3 \times 10^{-9}$ (see Belloche et al. 2009), in reasonably good agreement with the range of observed values in W51 e2 and 
SgrB2 N: $(3.6-10) \times 10^{-9}$. In addition, unlike the gas-phase model, the $\mathrm{HCOOH}$ abundance predicted by the grain-surface model is much closer to the observed values. The $[\mathrm{HCOOH} / \mathrm{EF}]$ ratio predicted by the surface chemistry models is $\sim 2$, in reasonable agreement with the values of $\sim 0.4-1$ found in W51 e2 and SgrB2 N. The abundances of the other COMs (i.e., MF and ET) predicted by the grain-surface model $(10-17) \times 10^{-8}$ are also consistent with those observed in W51 e 2 and SgrB2 N (Table 3).

Moreover, the excitation temperatures derived for $\mathrm{EF}$ in W51, SgrB2 and Orion KL (in the range 78-135 K; this work; Belloche et al. 2009; and Tercero et al. 2013) are in good agreement with the temperature associated with the peak gas-phase molecular abundance of the Garrod et al. model $(110 \mathrm{~K}$, see Table 15 of Belloche et al. 2009), further supporting the formation of EF on dust grains and subsequent evaporation to the gas phase.

Therefore, we conclude that $\mathrm{EF}$ and the other COMs detected in massive star-forming cores are likely formed on dust grains and then desorbed to the gas phase.

\section{Summary and conclusions}

We report on the detection of the two conformers (trans and gauche) of ethyl formate towards the W51 e 2 hot molecular core. Our LTE line profile analysis indicates that ethyl formate has an excitation temperature of $78 \pm 10 \mathrm{~K}$ and that both conformers have the same column density of $(2.0 \pm 0.3) \times 10^{16} \mathrm{~cm}^{-2}$, which yields to an abundance of $1.0 \times 10^{-8}$. We have discussed the formation of this complex species in the context of the two available competing chemical models based on dust grain surface and gasphase chemistry. We have derived the abundances of ethyl formate, other related complex molecular species and proposed precursors found in star-forming regions, and compared them with the predictions of chemical models. We find that complex organic molecules in hot cores are likely formed on the surface of dust grains and desorbed subsequently to the gas phase at higher temperatures.

Although the current data support grain-surface formation rather than gas-phase chemistry, it is clear that more detections of EF in a larger sample of astronomical sources, interferometric maps to better estimate the sizes of the emitting cores and thus the molecular abundances, and more laboratory experiments and theoretical models are needed to draw firm conclusions on the relative role of surface and gas-phase chemistry in the formation of EF and chemically related COMs.

Acknowledgements. This work was partly supported by the Italian Ministero dell'Istruzione, Università e Ricerca through the grant Progetti Premiali 2012 - iALMA. V.M.R. sincerely thanks Vianney Taquet for sharing the results of their gas-phase model before its publication, and Adam Ginsburg for the estimates of the W51 e2 hydrogen column density. J.M.-P. acknowleges partial support by the MINECO under grants AYA2010-2169-C04-01, FIS2012-39162-C06-01, ESP2013-47809-C03-01 and ESP2015-65597-C4-1.
P.C. acknowledges the financial support of the European Research Council (ERC; project PALs 320620).

\section{References}

Balucani, N., Ceccarelli, C., \& Taquet, V. 2015, MNRAS, 449, L16

Belloche, A., Garrod, R. T., Müller, H. S. P., et al. 2009, A\&A, 499, 215

Beltrán, M. T., Codella, C., Viti, S., Neri, R., \& Cesaroni, R. 2009, ApJ, 690, L93

Bisschop, S. E., Jørgensen, J. K., van Dishoeck, E. F., \& de Wachter, E. B. M. 2007, A\&A, 465, 913

Brown, R. D., Crofts, J. G., Godfrey, P. D., et al. 1975, ApJ, 197, L29

Carroll, P. B., McGuire, B. A., Blake, G. A., et al. 2015, ApJ, 799, 15

Cazaux, S., Tielens, A. G. G. M., Ceccarelli, C., et al. 2003, ApJ, 593, L51

Charnley, S. B., Kress, M. E., Tielens, A. G. G. M., \& Millar, T. J. 1995, ApJ, 448, 232

Demaison. J., Boucher, D., Burie, J., \& Dubrulle, A. 1984, Z. Naturforsch. A, 39,560

Demyk, K., Wlodarczak, G., \& Carvajal, M. 2008, A\&A, 489, 589

Favre, C., Despois, D., Brouillet, N., et al. 2011, A\&A, 532, A32

Fontani, F., Pascucci, I., Caselli, P., et al. 2007, A\&A, 470, 639

Garrod, R. T., \& Herbst, E. 2006, A\&A, 457, 927

Garrod, R. T., Weaver, S. L. W., \& Herbst, E. 2008, ApJ, 682, 283

Geppert, W. D., Hamberg, M., Thomas, R. D., et al. 2006, Faraday Discussions, 133,177

Hama, T., \& Watanabe, N. 2013, Chem. Rev., 113, 8783

Hamberg, M., Österdahl, F., Thomas, R. D., et al. 2010a, A\&A, 514, A83

Hamberg, M., Zhaunerchyk, V., Vigren, E., et al. 2010b, A\&A, 522, A90

Horn, A., Møllendal, H., Sekiguchi, O., et al. 2004, ApJ, 611, 605

Ikeda, M., Ohishi, M., Nummelin, A., et al. 2001, ApJ, 560, 792

Ioppolo, S., Cuppen, H. M., van Dishoeck, E. F., \& Linnartz, H. 2011, MNRAS, 410, 1089

Kalenskii, S. V., \& Johansson, L. E. B. 2010, Astron. Rep., 54, 1084

Kurtz, S., Cesaroni, R., Churchwell, E., Hofner, P., \& Walmsley, C. M. 2000, Protostars and Planets IV, 299

Liu, S.-Y., Mehringer, D. M., \& Snyder, L. E. 2001, ApJ, 552, 654

Lykke, J. M., Favre, C., Bergin, E. A., \& Jørgensen, J. K. 2015, A\&A, 582, A64

Medvedev, I. R., De Lucia, F. C., \& Herbst, E. 2009, ApJS, 181, 433

Menten, K. M., Reid, M. J., Forbrich, J., \& Brunthaler, A. 2007, A\&A, 474, 515

Millar, T. J., Bennett, A., Rawlings, J. M. C., Brown, P. D., \& Charnley, S. B. 1991, A\&AS, 87, 585

Peng, Z., Shlykov, S., Van Alsenoy, C., Geise, H. J., \& Van der Veken, B. 1995, J. Phys. Chem., 99, 10201

Remijan, A., Snyder, L. E., Liu, S.-Y., Mehringer, D., \& Kuan, Y.-J. 2002, ApJ, 576,264

Requena-Torres, M. A., Martín-Pintado, J., Martín, S., \& Morris, M. R. 2008, ApJ, 672, 352

Riveros, J. M., \& Wilson, E. B. 1967, J. Chem. Phys., 46, 4605

Rivilla, V. M., Fontani, F., Beltrán, M. T., et al. 2016, ApJ, 826, 161

Rivilla, V. M., Beltrán, M. T., Cesaroni, R., et al. 2017, A\&A, 598, A59

Schmiedeke, A., Schilke, P., Möller, T., et al. 2016, A\&A, 588, A143

Scott, P. F. 1978, MNRAS, 183, 435

Snyder, L. E., Lovas, F. J., Hollis, J. M., et al. 2005, ApJ, 619, 914

Taquet, V., Wirström, E. S., \& Charnley, S. B. 2016, ApJ, 821, 46

Tercero, B., Kleiner, I., Cernicharo, J., et al. 2013, ApJ, 770, L13

Tercero, B., Cernicharo, J., López, A., et al. 2015, A\&A, 582, L1

Tielens, A. G. G. M., \& Whittet, D. C. B. 1997, in IAU Symp. 178, ed. E. F. van Dishoeck, 45

Vigren, E., Hamberg, M., Zhaunerchyk, V., et al. 2010, ApJ, 722, 847

Woods, P. M., Kelly, G., Viti, S., et al. 2012, ApJ, 750, 19

Xu, Y., Reid, M. J., Menten, K. M., et al. 2009, ApJ, 693, 413

Zhang, Q., \& Ho, P. T. P. 1997, ApJ, 488, 241

Zhang, Q., Ho, P. T. P., \& Ohashi, N. 1998, ApJ, 494, 636 


\section{Appendix A: Spectra of MF, ET,}

\section{and trans-HCOOH}

We present some selected unblended transitions of MF, ET and t-HCOOH in Figs. A.1-A.3, and Tables A.1-A.3.
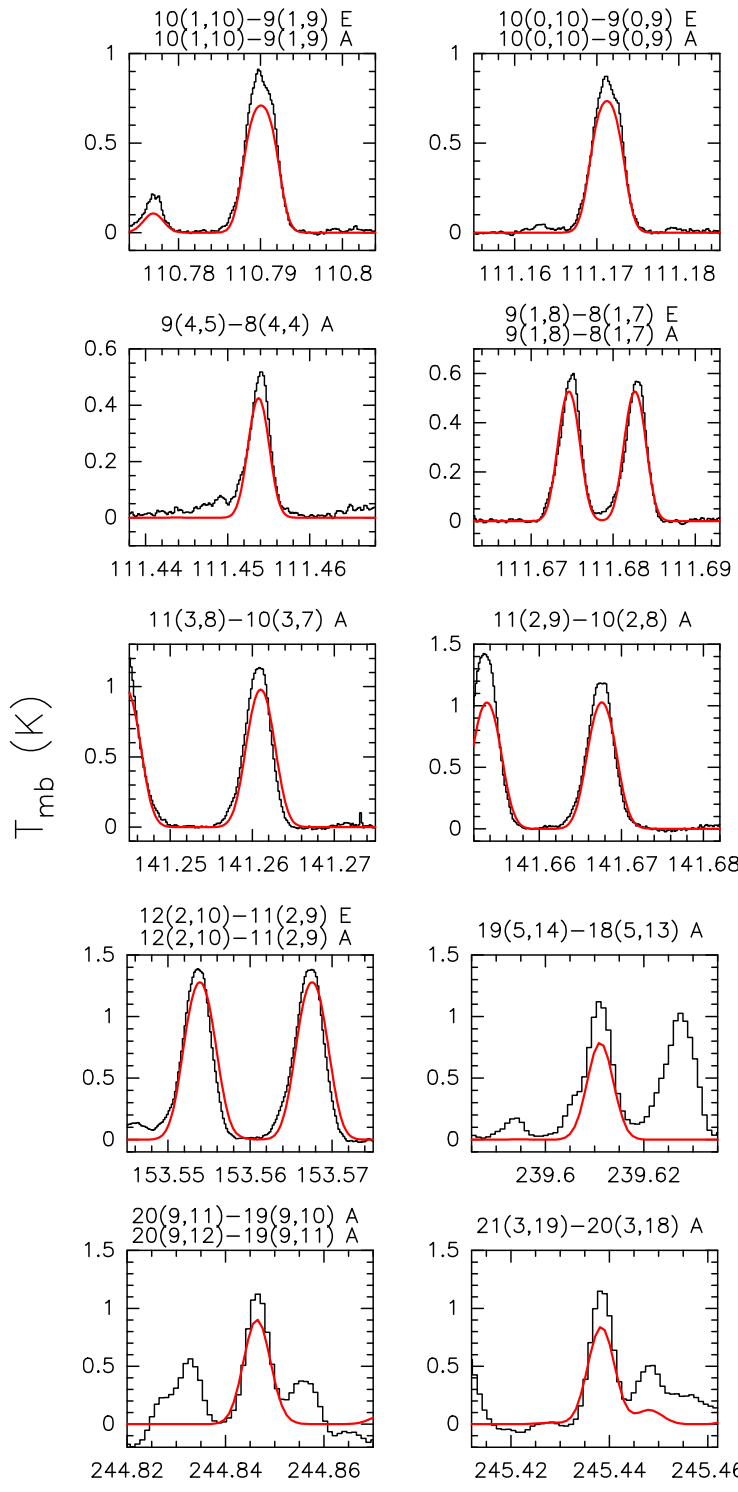

$9(1,8)-8(1,7) \mathrm{E}$
$9(1,8)-8(1,7) \mathrm{A}$
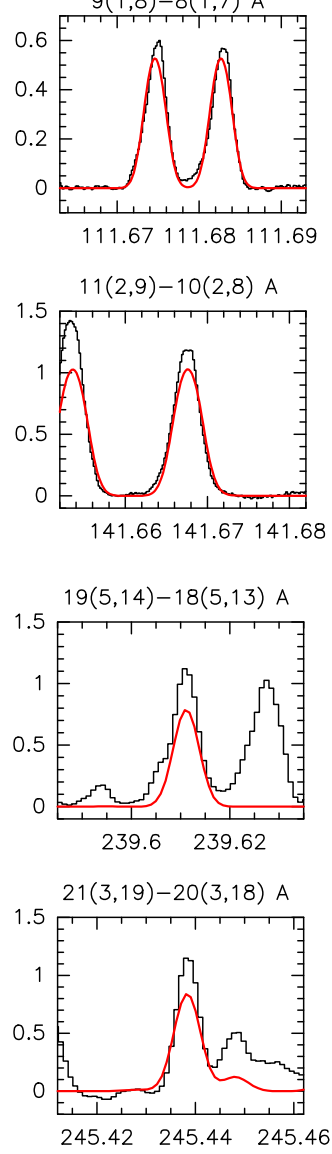

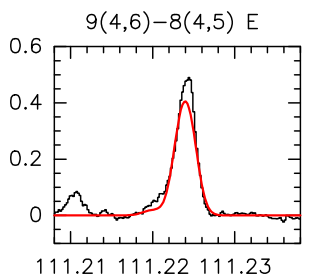

$11(5,6)-10(5,5) \mathrm{E}$
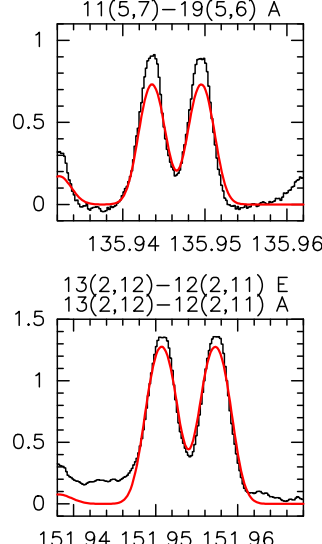

151.94151 .95151 .96
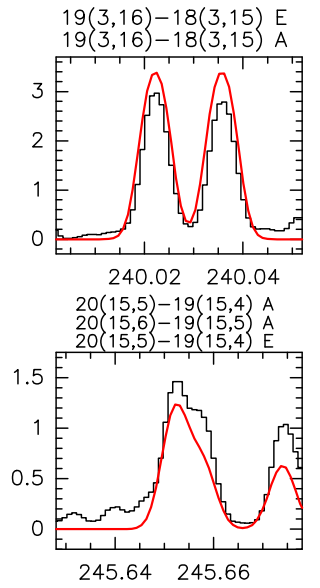

$20(13,7)-19(13,6) \mathrm{E}$ $20(13,8)-19(13,7)$ A $20(13,7)-19(13,6) A$ $20(13,8)-19(13,7) E$
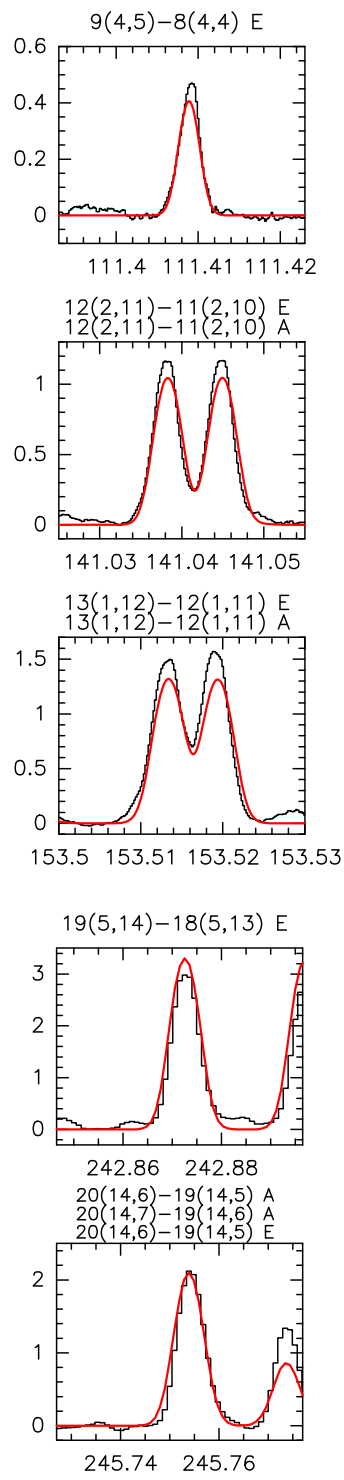

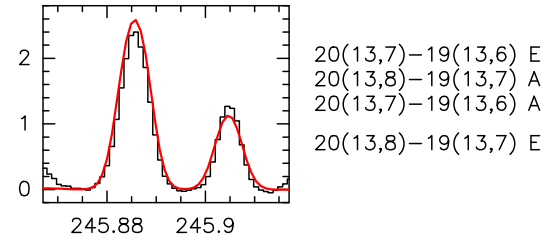

Frequency $(\mathrm{GHz})$

Fig. A.1. Selected unblended transitions of MF (see Table A.1) with LTE fit (red line). 
V. M. Rivilla et al.: Detection and formation of ethyl formate in the W51 e2 hot molecular core
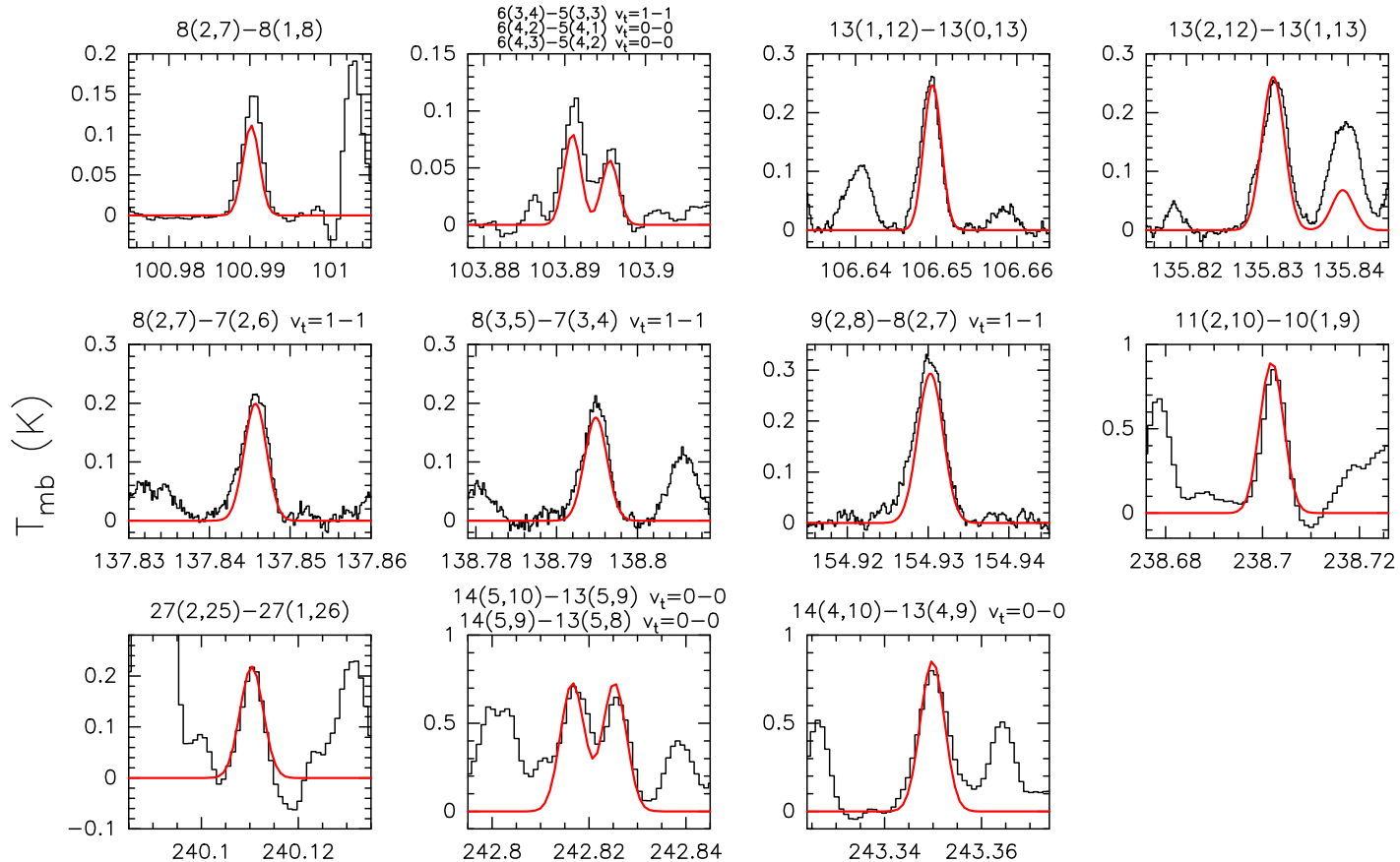

$238.68 \quad 238.7 \quad 238.72$

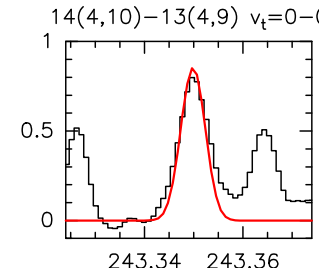

Frequency $(\mathrm{GHz})$

Fig. A.2. Selected unblended transitions of ET (see Table A.2) with LTE fit (red line).
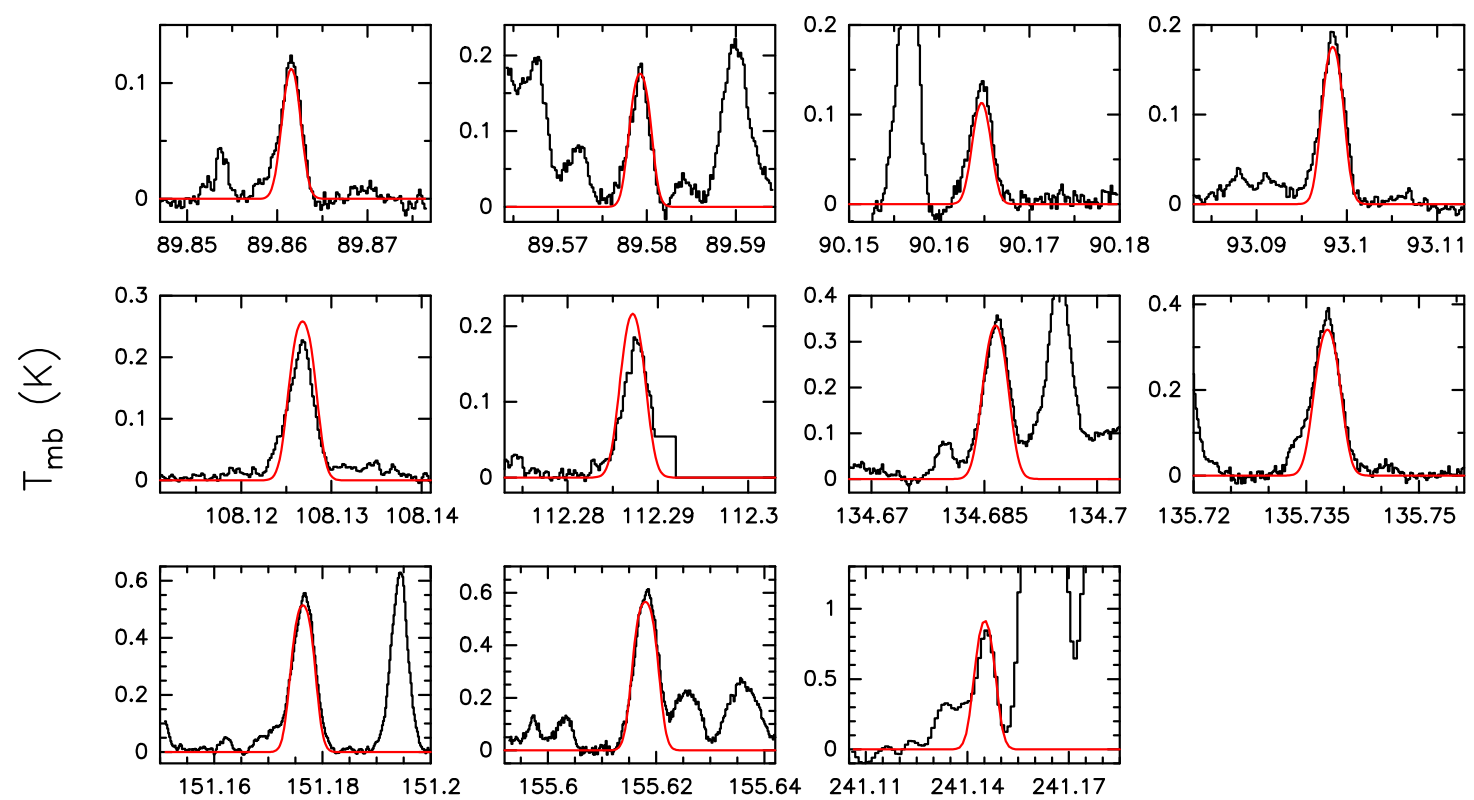

Frequency $(\mathrm{GHz})$

Fig. A.3. Selected unblended transitions of t-HCOOH (see Table A.3) with LTE fit (red line). 
Table A.1. Selected unblended transitions of MF.

\begin{tabular}{|c|c|c|c|}
\hline $\begin{array}{l}\text { Frequency } \\
(\mathrm{GHz})\end{array}$ & Transition & $\begin{array}{l}E_{\text {up }} \\
(\mathrm{K})\end{array}$ & 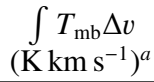 \\
\hline 110.788664 & $v=0,10(1,10)-9(1,9) \mathrm{E}$ & 30 & 5.0 \\
\hline 110.790526 & $v=0,10(1,10)-9(1,9) \mathrm{A}$ & 30 & 5.0 \\
\hline 111.169903 & $v=0,10(0,10)-9(0,9) \mathrm{E}$ & 30 & 5.0 \\
\hline 111.171634 & $v=0,10(0,10)-9(0,9) \mathrm{A}$ & 30 & 5.0 \\
\hline 111.223491 & $v=0,9(4,6)-8(4,5) \mathrm{E}$ & 37 & 3.5 \\
\hline 111.408412 & $v=0,9(4,5)-8(4,4) \mathrm{E}$ & 37 & 3.5 \\
\hline 111.453300 & $v=0,9(4,5)-8(4,4) \mathrm{A}$ & 37 & 3.7 \\
\hline 111.674131 & $v=0,9(1,8)-8(1,7) \mathrm{E}$ & 28 & 4.7 \\
\hline 111.682189 & $v=0,9(1,8)-8(1,7) \mathrm{A}$ & 28 & 4.7 \\
\hline 135.942980 & $v=0,11(5,7)-10(5,6) \mathrm{A}$ & 56 & 6.4 \\
\hline 135.948977 & $v=0,11(5,6)-10(5,5) \mathrm{E}$ & 56 & 6.4 \\
\hline 141.037702 & $v=0,12(2,11)-11(2,10) \mathrm{E}$ & 47 & 9.6 \\
\hline 141.044354 & $v=0,12(2,11)-11(2,10) \mathrm{A}$ & 47 & 9.6 \\
\hline 141.260421 & $v=0,11(3,8)-10(3,7) \mathrm{A}$ & 46 & 8.9 \\
\hline 141.667012 & $v=0,11(2,9)-10(2,8) \mathrm{A}$ & 43 & 9.4 \\
\hline 151.950079 & $v=0,13(2,12)-12(2,11) \mathrm{E}$ & 55 & 11.9 \\
\hline 151.956625 & $v=0,13(2,12)-12(2,11) \mathrm{A}$ & 55 & 11.9 \\
\hline 153.512752 & $v=0,13(1,12)-12(1,11) \mathrm{E}$ & 55 & 12.2 \\
\hline 153.518739 & $v=0,13(1,12)-12(1,11) \mathrm{A}$ & 55 & 12.2 \\
\hline 153.553231 & $v=0,12(2,10)-11(2,9) \mathrm{E}$ & 51 & 11.9 \\
\hline 153.566920 & $v=0,12(2,10)-11(2,9) \mathrm{A}$ & 51 & 11.9 \\
\hline 239.610154 & $v=1,19(5,14)-18(5,13) \mathrm{A}$ & 317 & 6.2 \\
\hline 240.021140 & $v=0,19(3,16)-18(3,15) \mathrm{E}$ & 122 & 32.6 \\
\hline 240.034673 & $v=0,19(3,16)-18(3,15) \mathrm{A}$ & 122 & 32.6 \\
\hline 242.871569 & $v=0,19(5,14)-18(5,13) \mathrm{E}$ & 130 & 31.0 \\
\hline 244.845337 & $v=1,20(9,12)-19(9,11) \mathrm{A}$ & 365 & 3.8 \\
\hline 244.845337 & $v=1,20(9,11)-19(9,10) \mathrm{A}$ & 365 & 3.8 \\
\hline 245.437310 & $v=1,21(3,19)-20(3,18) \mathrm{A}$ & 327 & 6.9 \\
\hline 245.651213 & $v=0,20(15,5)-19(15,4) \mathrm{A}$ & 273 & 5.1 \\
\hline 245.651213 & $v=0,20(15,6)-19(15,5) \mathrm{A}$ & 273 & 5.1 \\
\hline 245.656780 & $v=0,20(15,5)-19(15,4) \mathrm{E}$ & 273 & 5.1 \\
\hline 245.752266 & $v=0,20(14,6)-19(14,5) \mathrm{A}$ & 254 & 7.1 \\
\hline 245.752266 & $v=0,20(14,7)-19(14,6) \mathrm{A}$ & 254 & 7.1 \\
\hline 245.754100 & $v=0,20(14,6)-19(14,5) \mathrm{E}$ & 254 & 7.1 \\
\hline 245.883179 & $v=0,20(13,7)-19(13,6) \mathrm{E}$ & 236 & 9.4 \\
\hline 245.885243 & $v=0,20(13,8)-19(13,7) \mathrm{A}$ & 236 & 9.4 \\
\hline 245.885243 & $v=0,20(13,7)-19(13,6) \mathrm{A}$ & 236 & 9.4 \\
\hline 245.903680 & $v=0,20(13,8)-19(13,7) \mathrm{E}$ & 236 & 9.4 \\
\hline
\end{tabular}

Notes. ${ }^{(a)}$ From the LTE model fit (see text).
Table A.2. Selected unblended transitions of ET.

\begin{tabular}{cccc}
\hline \hline $\begin{array}{c}\text { Frequency } \\
(\mathrm{GHz})\end{array}$ & Transition $^{b}$ & $\begin{array}{c}E_{\mathrm{up}} \\
(\mathrm{K})\end{array}$ & $\begin{array}{c}\int T_{\mathrm{mb}} \Delta v \\
\left(\mathrm{~K} \mathrm{~km} \mathrm{~s}^{-1}\right)^{a}\end{array}$ \\
\hline 100.990102 & $8(2,7)-8(1,8)$ & 35 & 0.8 \\
103.890904 & $6(4,3)-5(4,2) v_{\mathrm{t}}=0-0$ & 94 & 0.3 \\
103.890904 & $6(4,2)-5(4,1) v_{\mathrm{t}}=0-0$ & 94 & 0.3 \\
103.895533 & $6(3,4)-5(3,3) v_{\mathrm{t}}=1-1$ & 90 & 0.4 \\
106.649479 & $13(1,12)-13(0,13)$ & 79 & 1.9 \\
135.830641 & $13(2,12)-13(1,13)$ & 81 & 2.0 \\
137.845614 & $8(2,7)-7(2,6) v_{\mathrm{t}}=1-1$ & 96 & 1.5 \\
138.794762 & $8(3,5)-7(3,4) v_{\mathrm{t}}=1-1$ & 103 & 1.3 \\
154.930188 & $9(2,8)-8(2,7) v_{\mathrm{t}}=1-1$ & 104 & 2.2 \\
238.70176 & $11(2,10)-10(1,9)$ & 60 & 6.9 \\
240.110238 & $27(2,25)-27(1,26)$ & 327 & 1.6 \\
242.816446 & $14(5,10)-13(5,9) v_{\mathrm{t}}=0-0$ & 175 & 5.5 \\
242.825099 & $14(5,9)-13(5,8) v_{\mathrm{t}}=0-0$ & 175 & 5.5 \\
243.349723 & $14(4,10)-13(4,9) v_{\mathrm{t}}=0-0$ & 164 & 6.5 \\
\hline
\end{tabular}

Notes. ${ }^{(a)}$ From the LTE model fit (see text). ${ }^{(b)}$ The transitions of the gauche state of ethanol are designated with $v_{\mathrm{t}}=0($ gauche +$)$ and $v_{\mathrm{t}}=1$ (gauche - ), while the transitions without $v$ number correspond to the trans state.

Table A.3. Selected unblended transitions of t-HCOOH.

\begin{tabular}{cccc}
\hline \hline $\begin{array}{c}\text { Frequency } \\
(\mathrm{GHz})\end{array}$ & Transition & $\begin{array}{c}E_{\mathrm{up}} \\
(\mathrm{K})\end{array}$ & $\begin{array}{c}\int \mathrm{T}_{\mathrm{mb}} \Delta v \\
\left(\mathrm{~K} \mathrm{~km} \mathrm{~s}^{-1}\right)^{a}\end{array}$ \\
\hline 89.57917 & $4(0,4)-3(0,3)$ & 10.8 & 1.7 \\
89.86148 & $4(2,3)-3(2,2)$ & 23.5 & 1.0 \\
90.16463 & $4(2,2)-3(2,1)$ & 23.5 & 1.0 \\
93.09836 & $4(1,3)-3(1,2)$ & 14.4 & 1.7 \\
108.12670 & $5(1,5)-4(1,4)$ & 18.8 & 2.5 \\
112.28712 & $5(2,4)-4(2,3)$ & 28.9 & 1.9 \\
134.68637 & $6(2,5)-5(2,4)$ & 35.4 & 3.1 \\
135.73770 & $6(2,4)-5(2,3)$ & 35.5 & 3.1 \\
151.17628 & $7(1,7)-6(1,6)$ & 32.3 & 5.3 \\
155.61788 & $7(0,7)-6(0,6)$ & 30.0 & 6.0 \\
241.14633 & $11(0,11)-10(0,10)$ & 70.2 & 8.4 \\
\hline
\end{tabular}

Notes. ${ }^{(a)}$ From the LTE model fit (see text). 